Center (Madrid, Spain) during the validation study of the OSTRC-H2 Spanish questionnaire.

Patients (or Participants) Fifty-four elite athletes (swimming, waterpolo and artistic swimming), from 12 to 18 years old.

Interventions (or Assessment of Risk Factors) During 10 consecutive Sundays, athletes completed online the Spanish OSTRC-H2 questionnaire and 2 questions proposed by the Fédération Internationale de Natation about time-loss of activity. Athletes with musculoskeletal complaints were sent by coaches to Physiotherapy consultations, carried out three times a week.

Main Outcome Measurements Injured athletes (time-loss injury or 'all complaints') and floor and ceiling effects of severity scores (OSTRC-H2 score and total and partial self-reported days/week of time-loss).

Results Thirty-six athletes reported injury complaints through the OSTRC-H2 questionnaire. Twenty reported at least one time-loss injury. Physiotherapy consultations detected $41 \%$ of the athletes with injury complaints and $35 \%$ of those with time-loss injuries. All requests for consultation were attended. Injury severity measures showed different floor and ceiling effects $(84 \%$ and $4 \%$ for total time-loss; $71 \%$ and $12 \%$ for partial time-loss; $6 \%$ and $3 \%$ for OSTRC-H2 score).

Conclusions The OSTRC-H2 Spanish questionnaire has shown greater ability to detect young injured athletes than a method based on physiotherapy consultations. Moreover, OSTRC-H2 severity score has shown greater comprehensiveness than a self-reported time-loss approach.

\section{EPIDEMIOLOGY OF YOUTH INJURIES ACROSS SEVEN SPORTS AT A SINGLE COLLEGE IN ENGLAND}

${ }^{1,4}$ Craig Barden, ${ }^{5}$ Ken Quarrie, ${ }^{1,2}$ Carly McKay, ${ }^{1,3}$ Keith Stokes. ${ }^{1}$ Department for Health, University of Bath, Bath, BA2 7AY, UK., Bath, UK; ${ }^{2}$ Centre for Motivation and Health Behaviour Change, University of Bath, Bath, BA2 7AY, UK., Bath, UK; ${ }^{3}$ Rugby Football Union, Twickenham, TW2 7BA, UK, London, UK; ${ }^{4}$ SGS Sport, South Gloucestershire and Stroud College, Bristol, BS34 8LP, UK, Bristol, UK; ${ }^{5}$ New Zealand Rugby, Wellington, New Zealand, Wellington, New Zealand

\subsection{6/bjsports-2021-IOC.276}

Background There is increasing drive to assess injury risk in youth sports, given the potential health risks associated with participation. However, many studies focus upon a single sport and comparison between studies is often difficult due to varied injury definitions and methodologies.

Objective To investigate overall injury risk in youth collegiate sports at a single site using consistent data collection methods and injury definitions, and to compare between sports.

Design Retrospective cohort study (2015-2019).

Setting One elite sports college in England.

Participants Under-17 to under-19-year-old athletes enrolled in seven college teams [male American football, basketball, football, rugby league, rugby union; female football and rugby union].

Main outcome measures Injuries ( $>24$-hour time loss) and match exposure were recorded on a standardised report form by college medical staff. Descriptive statistics [percentages, median, range, 95\% confidence-intervals (95\% CI)] and injury incidence (per 1000 player-match-hours) are reported.
Results In total, 322 injuries were sustained by 240 athletes in 10,273 hours of match exposure. Overall injury incidence was $31.3 / 1000 \mathrm{~h}$ (95\% CI 28-35) with a median severity of 23 days lost (1-427). Lower limb (52\%) injuries were most common, followed by head/neck (26\%), whilst $60 \%$ of injuries resulted from player contact. American football had the greatest injury incidence $(85.9 / 1000 \mathrm{~h} ; 95 \%$ CI 61-120). Female rugby union $(53.4 / 1000 \mathrm{~h} ; 95 \%$ CI $37-76)$, male rugby union (51.2/1000h; 95\% CI 43-61) and basketball (42.9/1000h; $95 \%$ CI 25-72) had a substantially greater injury incidence than male football $(15.9 / 1000 \mathrm{~h} ; 95 \%$ CI 13-20) and female football $(21.3 / 1000 \mathrm{~h} ; 95 \%$ CI 14-33), but not rugby league (23.7/1000h; 95\% CI 12-46).

Conclusions Common injury characteristics were identified across all sports. Despite being non-contact, basketball had higher injury risk than three other sports, which warrants further investigation. This study highlights the benefits of consistent data collection methods and injury definitions across sports, particularly in youth settings.

\section{YOUTH VOLLEYBALL, BASKETBALL AND FUTSAL ATHLETES PERFORMANCE ON Y-TEST OVER THE SPORTS SEASON}

${ }^{1}$ Renato de Paula da Silva, ${ }^{1}$ Ellen Exmalte de Castro Aguiar, ${ }^{2}$ Leandro Cézar Garcia, ${ }^{2}$ Felipe Ribeiro Pereira, ${ }^{2}$ Silvanio Miranda Signoretti Júnior, ${ }^{1}$ Renan Alves Resende, ${ }^{2}$ Natália FN Bittencourt. 'UFMG - Universidade Federal de Minas Gerais, Belo Horizonte, Brazil; ${ }^{2}$ Minas Tênis Clube, Belo Horizonte, Brazil

\subsection{6/bjsports-2021-IOC.277}

Background Musculoskeletal assessment is very important for injuries prevention and should be made in different moments of sports season. Dynamic lower limb stability is an essential parameter for a good performance, and it can be assessed by Y-Test.

Objective To compare Y-Test performance during 3 different moments of an entire season in young futsal, volleyball and basketball athletes of a Brazilian sports club.

Design Observation (prospective).

Setting One club facility in Brazil.

Patients (or Participants) 90 male athletes divided in: jumpers (volleyball and basketball, 64 subjects); runners (futsal, 26 subjects).

Interventions (or Assessment of Risk Factors) All subjects performed Y-Test, which involves reaches with contralateral leg the furthest distance while maintaining single-leg stance in three directions (anterior, posteromedial, and posterolateral) in three different moments of the year.

Main Outcome Measurements Asymmetry between legs in each direction and composite scores (average between normalized distances in each direction divided by leg length).

Results ANOVA repeated measures were used in statistical analysis $(\mathrm{p}<0,05)$. Regarding asymmetry, the results showed significant difference only in the posteromedial direction of jumpers group $(p=0,003)$, indicating an improvement throughout the season. Runners did not present any difference. In relation to composite scores, it was found a statistically significant difference in the non-dominant leg in runners' group ( $\mathrm{p}$ $=0,016)$, suggesting a decline of performance on Y-Test during the season. Moreover, the results found the dominant leg were bordering $\mathrm{p}$ value $(\mathrm{p}=0,06$ in jumpers and $\mathrm{p}=0,0502$ in 
runners), implying a decreased efficacy in jumpers and increased in runners.

Conclusions Throughout season neuromuscular adaptations occurred accordingly with sports gestures and this might explain the different results found between groups. Fatigue can be an important factor to explain the decrease of performance of runners. Sports with consecutive jumps maybe are related with the reduction of asymmetry, what does not happen between runners.

\section{INJURY BURDEN AND CHARACTERISTICS IN AESTHETIC SPORTS AMONG HIGH SCHOOL ADOLESCENTS}

1,2,3 Sheila Downie, ${ }^{1}$ Amanda M Black, ${ }^{1,2,4}$ Paul H Eliason, ${ }^{1,2,3,4,5,6}$ Carolyn Emery, ${ }^{1,2,3}$ Sarah J Kenny. 'Sport Injury Prevention Research Centre, Faculty of Kinesiology, University of Calgary, Calgary, Canada; ${ }^{2}$ Alberta Children's Hospital Research Institute, Cumming School of Medicine, University of Calgary, Calgary, Canada; ${ }^{3} O^{\prime} B r i e n$ Institute for Public Health, Cumming School of Medicine, University of Calgary, Calgary, Canada; ${ }^{4}$ Hotchkiss Brain Institute, Cumming School of Medicine, University of Calgary, Calgary, Canada; ${ }^{5}$ Community Health Sciences, Cumming School of Medicine, University of Calgary, Calgary, Canada; ${ }^{6}$ Department of Pediatrics, Cumming School of Medicine, University of Calgary, Calgary, Canada

\subsection{6/bjsports-2021-IOC.278}

Background Aesthetic sports require athletes to showcase extreme flexibility, aerial maneuvers and perform hard surface landings that may increase injury risk. However, very few studies have examined injury risk in this population.

Objectives To identify aesthetic sport injury prevalence among high school students and to describe the type, location and severity of injury in adolescents who practice aesthetic sports.

Design Cross-sectional study.

Setting High schools in Calgary area, Canada.

Participants Adolescent students $(\mathrm{n}=2029 ; 958$ male, 1048 female, 23 identified 'other'; ages 14-19 year) from 24 high schools.

Assessment of Risk Factors Self-reported participation in aesthetic sport (i.e., gymnastics, dance, figure skating) in previous one-year (based on top three sports for participation).

Main Outcome Measurements Self-reported injury (time loss/ medical attention), type, anatomical location, and severity. Proportions $[95 \%$ confidence intervals (CI)] were adjusted for cluster by school.

Results Among the 2029 students who completed the question about sport participation, 15\% (302/2029) participated in aesthetic sports (282 female, 20 males; dance (247/302; 82\%), gymnastics $(50 / 302 ; 16 \%)$, figure skating $(22 / 302 ; 7 \%)$. In the previous one-year, 74 females $(26.2 \%$; 95\% CI, 20.8-32.6) and 2 males (10.0\%; 95\% CI, 2.6-31.2) listed aesthetic sport injury as the most severe. Ankle (26.3\%; 95\% CI, 17.5-37.6), knee $(25.0 \%$; 95\% CI, 16.4-36.2), and back (9.2\%; 95\% CI 4.4-18.4) were the most common injury sites. Ligament sprains $(22.7 \%$; 95\% CI 14.4-33.7), muscle strains $(14.7 \%$; 95\%CI 8.2-24.9), and fractured bones $(12.0 \%$; 95\% CI 6.321.8) were most common injury types. Medical attention injury rate was 20.5/100 athletes/year and time-loss $>7$ days injury rate was 11.9/100 athletes/year.

Conclusions Aesthetic sport participation and injury rates among high school students are high. The most serious injuries reported were lower extremity injuries with a greater proportion of females reporting aesthetic sport injuries than males. Future research should focus on mitigation of lower extremity injuries among these high-risk aesthetic athletes.

\section{PREVALENCE AND BURDEN OF HEALTH PROBLEMS IN COMPETITIVE ADOLESCENT DISTANCE RUNNERS: A 6- MONTH PROSPECTIVE COHORT STUDY}

${ }^{1}$ Robert Mann, 2,3 Benjamin Clarsen, ${ }^{4}$ Carly McKay, ${ }^{4}$ Bryan Clift, ${ }^{1}$ Craig Williams, ${ }^{1}$ Alan Barker. 'Children's Health and Exercise Research Centre, University of Exeter, Exeter, UK; ${ }^{2}$ Department of Health Promotion, Norwegian Institute of Public Health, Oslo, Norway; ${ }^{3}$ Department of Sports Medicine, Norwegian School of Sports Sciences, Oslo, Norway; ${ }^{4}$ Department for Health, University of Bath, Bath, UK

\subsection{6/bjsports-2021-IOC.279}

Background Little is known about the overall health of adolescent distance runners.

Objective To describe all health problems (injuries and illnesses) in relation to type, location, incidence, prevalence, time loss, severity, and burden, in competitive adolescent distance runners in England.

Design Prospective observational study monitoring all health problems for 24-weeks between May and October (2019).

Setting Competitive adolescent distance runners (i.e., $800 \mathrm{~m}$ to $10,000 \mathrm{~m}$, including steeplechase) in England.

Patients (Or Participants) Distance runners (13-18 y) were invited to participate if they had achieved a top-50 performance in their age-group (U20, U17 and U15) during 2018. A total of 644 athletes were invited to take part, with 136 athletes (73 females) having enrolled and completed the study (lost to follow-up: $\mathrm{n}=7$ ).

Main Outcome Measurements The prevalence and burden of health problems was recorded using the Oslo Sports Trauma Research Center Questionnaire on Health Problems (OSTRC$\mathrm{H})$. The OSTRC-H was completed online, via Qualtrics, on a weekly basis.

Results A total of 363 health problems were registered during this study, including 213 injuries and 150 illnesses. At any time, 24\% [95\% Confidence Intervals (CI): 21-26\%] of athletes reported a health problem, with 11\% [95\% CI: 9-12\%] having experienced a health problem that had substantial negative impact on training and performance. Female athletes reported noticeably more illnesses, compared to male athletes, including higher prevalence, incidence, time loss, and severity. The most burdensome health problems, irrespective of sex, included lower leg, knee, and foot/toes injuries, alongside upper respiratory illnesses. The mean weekly prevalence of time loss was relatively low, regardless of health problem type or sex.

Conclusions Competitive adolescent distance runners are likely to be training and competing whilst concurrently experiencing health problems. These findings will support the development of injury and illness prevention measures.

\section{DOES THE FIFA 11+ SHOULDER INJURY PREVENTION PROGRAM REDUCE THE INCIDENCE OF UPPER EXTREMITY INJURIES AMONG SOCCER GOALKEEPERS? A RANDOMISED CONTROLLED TRIAL}

${ }^{1}$ Wesam Saleh A Al Attar, ${ }^{1,2}$ Mansour Abdullah Alshehri. 'Umm Al Qura University, Mecca, Saudi Arabia; ${ }^{2}$ University of Queensland, Brisbane, Australia

\section{$10.1136 /$ bjsports-2021-IOC.280}

Background Goalkeepers appear to be the players who are at most risk of sustaining a shoulder injury. Many researchers have reported that goalkeepers are more likely, than outfield players, to injure their upper limb. The FIFA $11+\mathrm{S}$ is 